\title{
Coupled radiative thermal and nonlinear stress analysis for thermal deformation in large space structures
}

\author{
Olive R. Stohlman* \\ NASA Langley Research Center, Hampton, VA
}

\begin{abstract}
Large space structures are capable of large thermal deformations in the space environment. A case of large-scale thermal deformation was observed in the analysis of the Near Earth Asteroid Scout solar sail, with predicted tip displacements of more than one meter in seven-meter booms. Experimental data supports the broad conclusions of the analysis, but shows poor agreement on the details of the thermal deformation. Prediction that is precise enough to drive engineering decisions will require coupled thermal-stress analysis with features that are not found in current multiphysics codes.

This paper describes a simple method for stepwise coupling between commercial nonlinear stress analysis software and radiative thermal analysis software. Results are presented for a round stainless steel tube, which is a common case in existing literature.
\end{abstract}

\section{Background}

This study of thermal deformation was originally motivated by the Near Earth Asteroid Scout (NEA Scout) mission (Figure 1). NEA Scout is a $6 \mathrm{U}$ Cubesat mission that will use a deployable solar sail to generate thrust. ${ }^{1}$ The sail membrane is deployed and shaped by a set of four booms, each nearly 7 meters long and made of Elgiloy, a cobalt alloy. The boom cross-section is the Triangular Rollable and Collapsible (TRAC) boom design. ${ }^{2}$

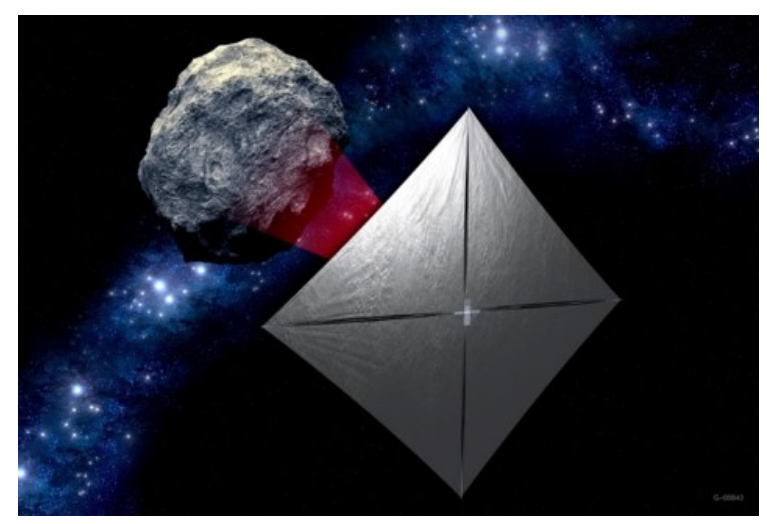

Figure 1: An artist's rendition of NEA Scout, which has a nominally flat sail surface.

Early thermal deformation analysis of the booms' performance indicated that they would experience large deformations in the space environment. Boom deformation is important to a solar sail because the boom shape determines the sail shape, and the sail shape determines the magnitude and direction of the solar radiation pressure thrust on the spacecraft. A large offset in the center of pressure or mass can produce a large disturbance torque on the spacecraft, and uncertainty in the thrust affects trajectory planning and control. Thin boom cross-sections can develop extreme thermal gradients in direct sunlight, and in TRAC booms these produce curvatures that do not obey a simple relationship with sun angle. This analysis is

\footnotetext{
* Research Engineer, Structural Dynamics Branch, NASA Langley Research Center. AIAA Member.
} 


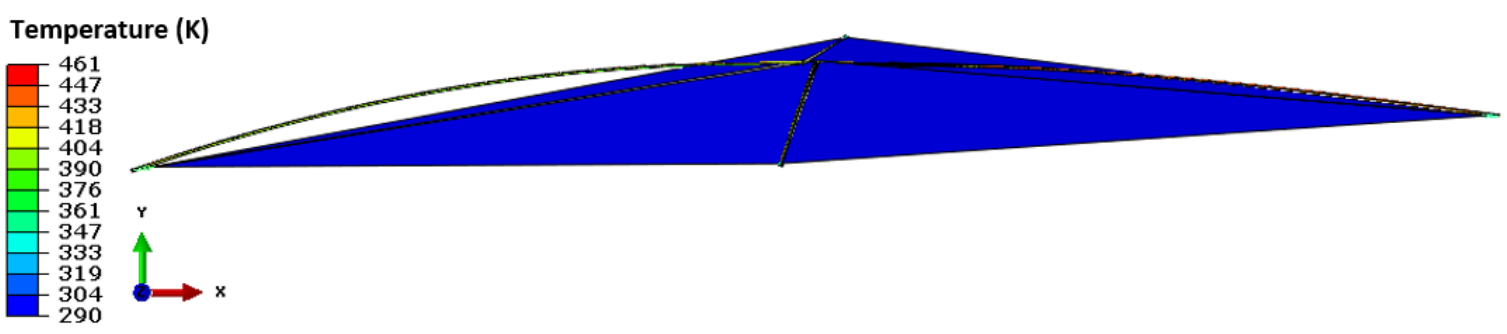

Figure 2: An early prediction of the distorted NEA Scout sail shape in the space thermal environment. ${ }^{3}$ The thermally distorted shape of the sail is far from the nominal flat square shape, leading to net torque on the spacecraft and a high margin of error in the spacecraft thrust.

discussed in an earlier paper, ${ }^{3}$ which also demonstrates the advantages of composite booms in reducing thermal distortion.

From this early analysis in the NEA Scout project, it was obvious that high-accuracy analysis of booms thermal deformation was impossible with existing software. The analysis procedure for NEA Scout was to calculate a boom temperature distribution in the thermal analysis software Thermal Desktop (TD) based on the undeformed booms, then apply these temperatures to a stress analysis model in the finite element stress analysis software Abaqus. Thermal analysis predicted thermal gradients of over $100 \mathrm{~K}$ within the boom cross-section. The stress analysis indicated large deformations, and particularly twisting deformations, in the booms under combined thermal and structural load. Figure 2 shows one example of a predicted deformed sail shape. These deformations would invalidate the thermal analysis, which had been based on the boom geometry at uniform temperature. Because there was no automated coupling of the boom temperature distribution and the boom deformation, the model results would have to be repeatedly passed between thermal and structural analysts to sequentially solve the evolving thermal and structural models, which was not practical. This work demonstrated a need for closely coupled thermal and stress analysis software, and suggested that this need will increase with increasing structural size.

Because the predicted boom shapes exceeded the tolerance for solar radiation pressure-induced torque on the spacecraft and higher-accuracy thermal-structural analysis was not available, the NEA Scout sail design was changed from a four-quadrant sail to a one-piece square sail. This allows the booms to be shaded by the sail membrane during normal operations, greatly reducing the thermal gradient on the shaded portions of the booms, and required design changes to the sail membrane and spool.

\section{A. Experimental background}

To justify the design changes that were made in response to the early analysis, and to confirm that these changes would solve the problem of thermal deformation in the metallic TRAC booms, the NEA Scout project suspended a 4-meter-long metallic TRAC boom vertically in a thermal vacuum chamber and subjected it to strong directional light from an infrared sunspot lamp. The experimental setup is shown in Figure 3(a). ${ }^{4}$

Three cameras, positioned as shown in Figure 3(b), recorded video of the boom's shape during the experiment. Boom tip displacements of tens of centimeters were easily identified in the video recordings, affirming that the magnitude of the boom deformation identified in analysis was correct. Some video frames are excerpted in Figure 4.

The boom's motion can be divided into three stages: Transition 1, where the lamps were turned on and the boom reached a briefly stable curved position, Torsional Motion, where the boom tip experienced a sustained torsional oscillation at $0.8 \mathrm{~Hz}$ for approximately 15 minutes, and Transition 2, where the oscillation ceased and the boom transitioned to a new stable curved state. These phases are captured in the temperature (Figure 5) and video results of the experiment in Figures 6 and 4. Because the experiment was not repeated, there are many possible explanations for the observed behaviors, including the vibration environment, thermal deformation of the support structure, and untracked changes in the thermal environment.

For the purposes of the NEA Scout project, only two demonstration results were important: that the boom experienced tens of centimeters of displacement at the tip under direct thermal load, and that the tip displacement was reduced to less than 3 centimeters when the boom was shaded by the solar sail membrane. 


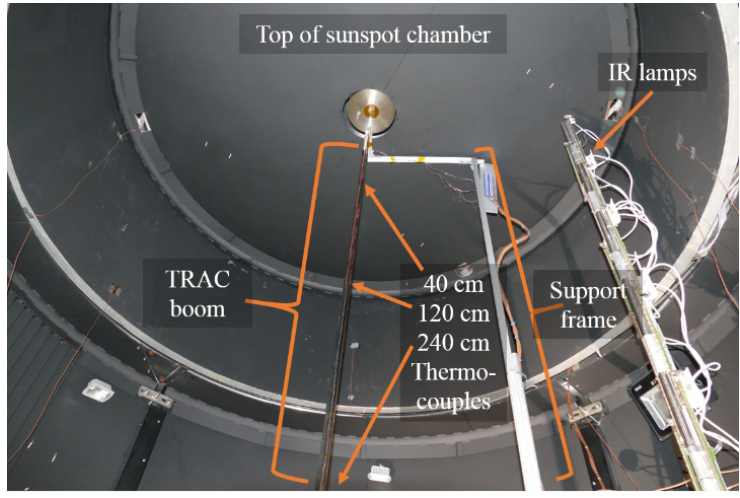

(a)

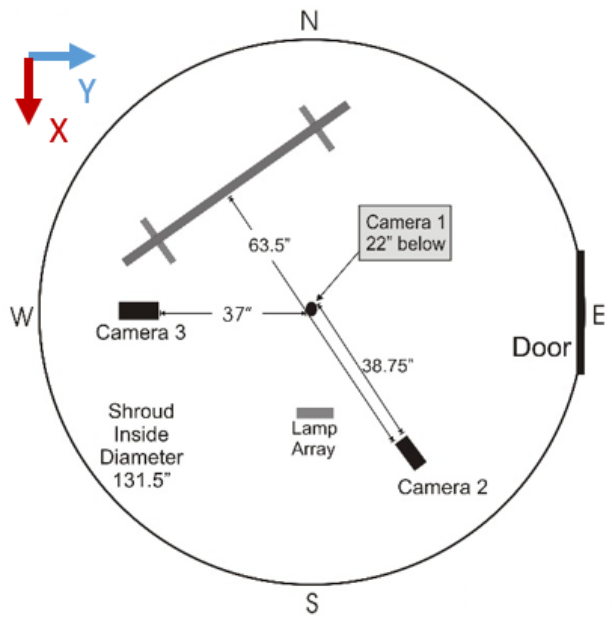

(b)

Figure 3: A photograph of the experimental setups (a) and a diagram of the camera positions (b). ${ }^{4}$
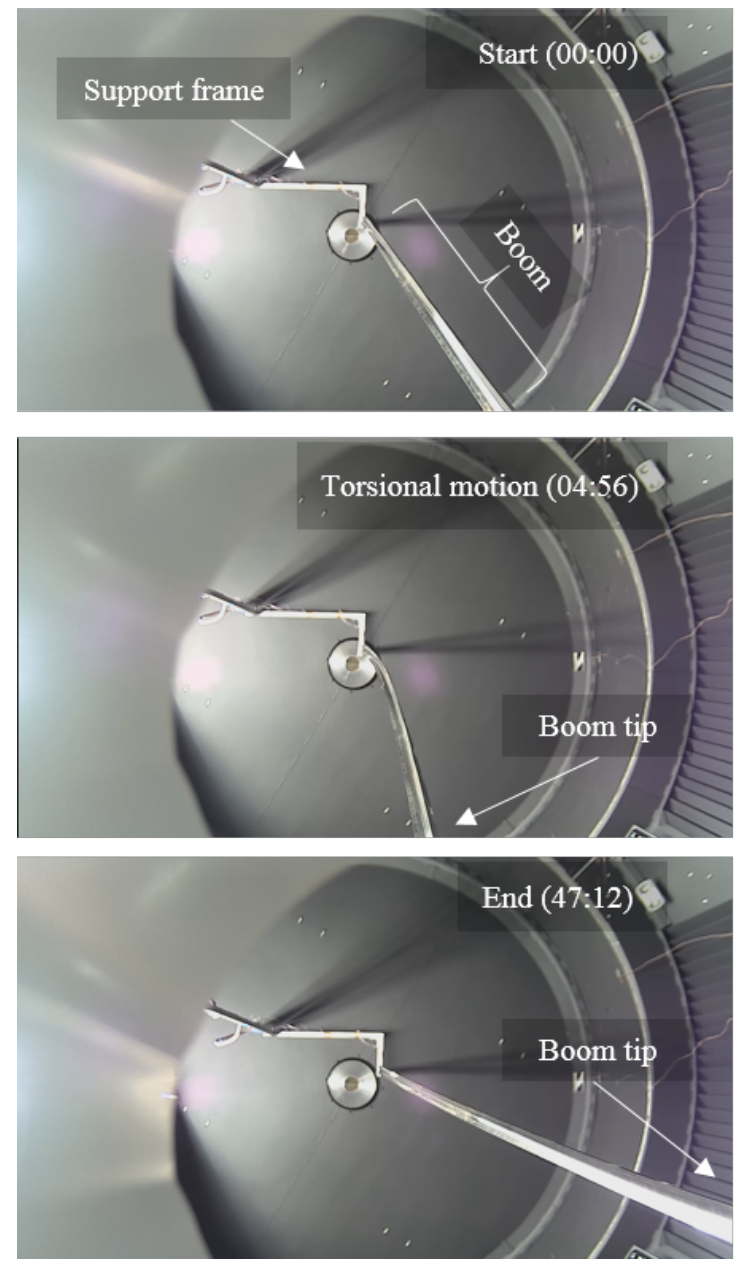

Figure 4: Experimental changes in the global boom shape were captured by Camera 1, below the boom, which used a wide-angle lens. ${ }^{4}$ 


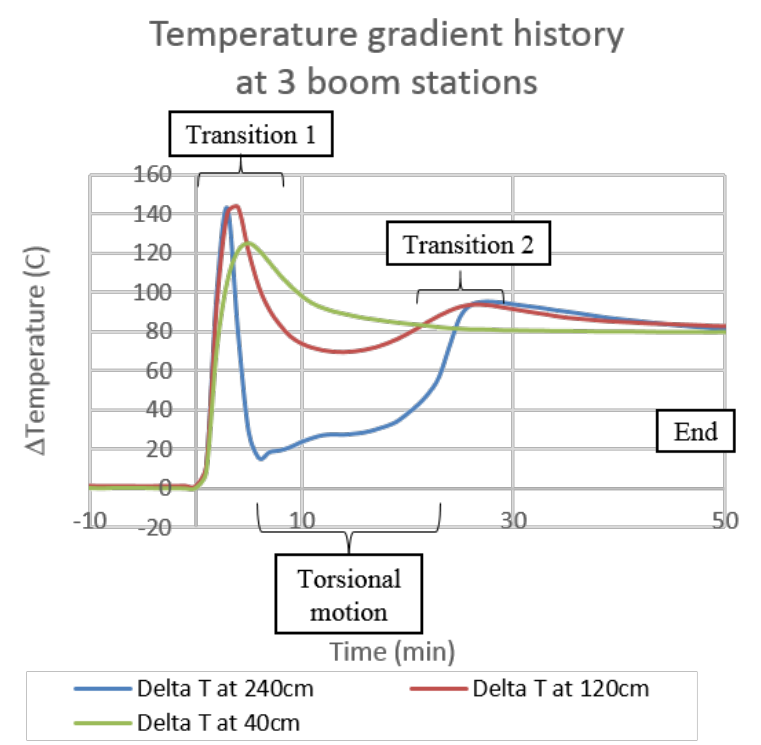

Figure 5: The boom motion is driven by temperature gradients within the boom cross-section. ${ }^{4}$

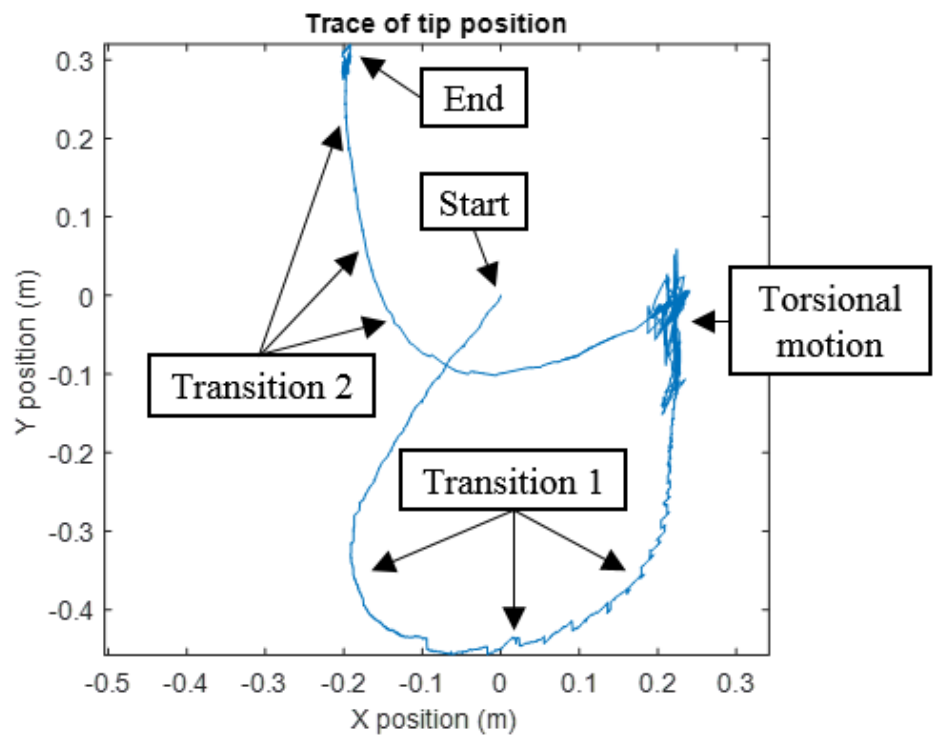

Figure 6: The boom tip, whose motion was derived from the captured video, traced out a complex shape under only these thermal loads. ${ }^{4}$ 
This confirmed the results of analysis and showed that the design solution would be effective. The more detailed results of the experiment were not a good match to analysis: the magnitude of the boom tip displacement was in agreement, but the direction and the path of the displacement were different. Further, the static analysis performed before the experiment was not equipped to predict oscillations or the rate of deformation.

All these un-captured effects were interesting, and raise the practical concern that larger future structures may present increasingly non-linear thermal-structural interactions. Even composite booms are subject to some degree of thermal distortion at large enough scales. It would be advantageous to be able to predict dynamic, large-scale thermal-structural interactions, and this prediction would necessarily involve radiative thermal analysis with orbital environment modeling as well as robust dynamic stress analysis.

Thermal and stress analysis have been coupled in various ways in the past. Blandino's work on dynamic thermal effects on a Hubble telescope boom ${ }^{5}$ is by far the closest to addressing this demand, and presents an elegant solution when it is possible to calculate the heat flux or temperature distribution in a boom through known relationships. The framework presented by Blandino was used as a guide in parts of this work. Hypersonic flight analysis may use far more sophisticated thermal-structural-fluid multiphysics approaches, ${ }^{6}$ but doesn't typically use the radiative heat transfer approaches that are important to large deployable space structures. Finally, a structure designed to be analytically assessed might be compatible with hand calculations and not require a numerical treatment of thermal flutter. ${ }^{7,8}$

Nonlinear finite element stress analysis and ray-tracing thermal-radiative analysis are each substantial challenges. They use fundamentally different solution techniques (particularly when an implicit stress analysis software, like Abaqus/Standard, is used). Coupling two existing pieces of software has advantages over creating new, purpose-built software, or adding ray-tracing and thermal environment capabilities to a stress analysis code: it allows analysts to take full advantage of the features available in mature commercial codes. The approach of this paper was to link these two pieces of software, handing off the geometry and temperatures between them at small time steps for sequential coupling.

\section{Modeling}

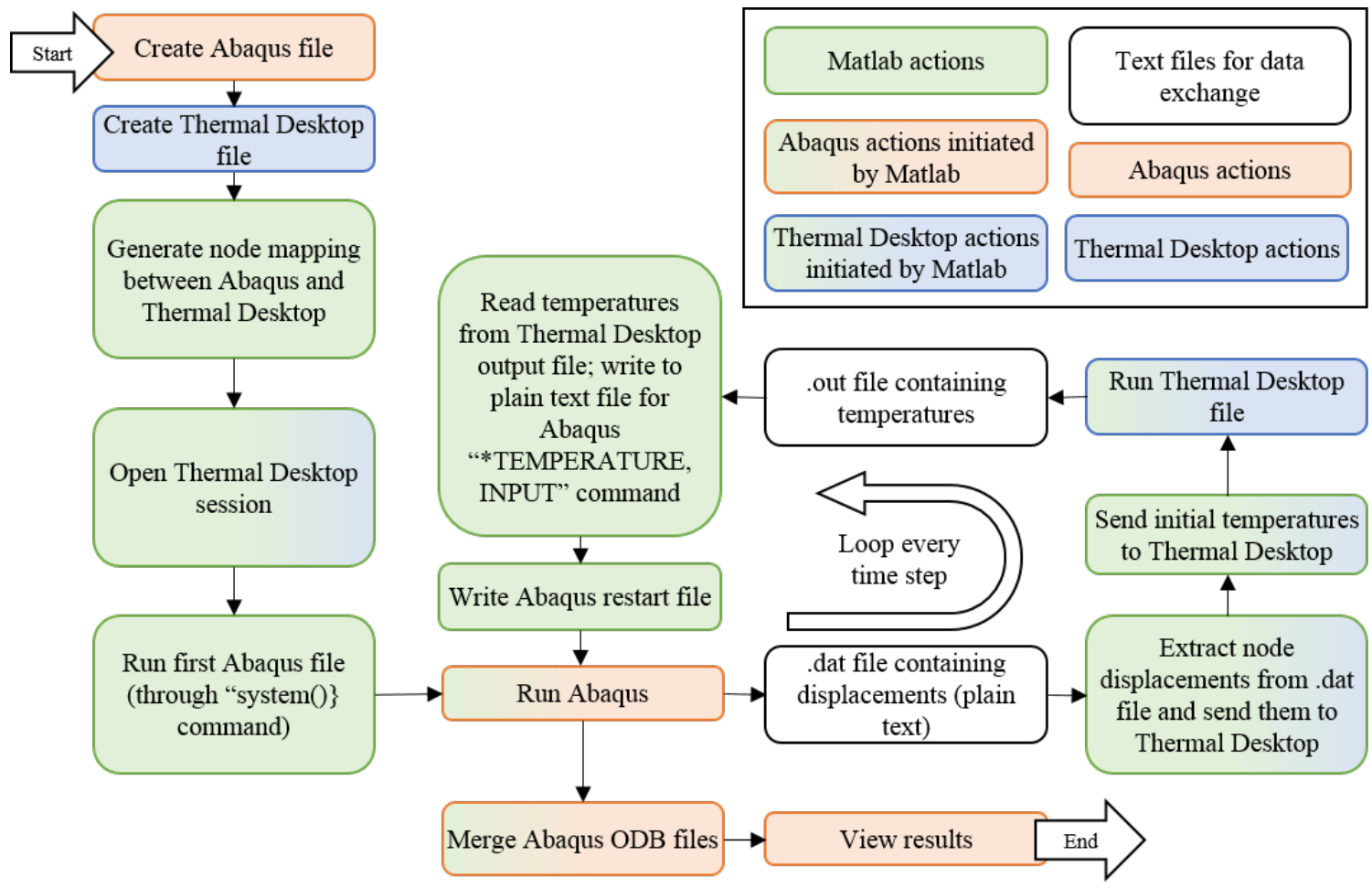

Figure 7: Flowchart of analysis procedure. 


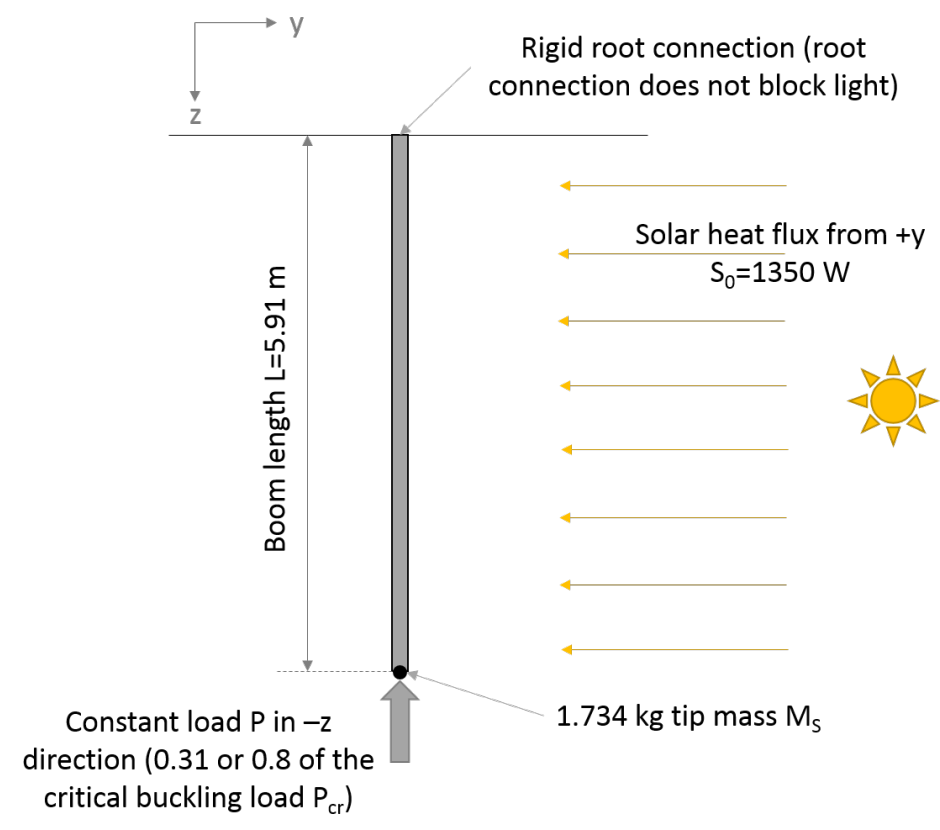

Figure 8: The boundaries and forces in the modeled boom scenario.

Abaqus and Thermal Desktop can be linked, stepwise, according to the strategy in Figure 7. A master program (in this case, Matlab) commands each of the two analysis programs and feeds results between them. Matlab was chosen for familiarity, but there are many other options for controlling the process. Whatever is used to control the two programs should be able to load the Microsoft .NET API that is provided with Thermal Desktop 6.0 (C\# is used in the Thermal Desktop API sample codes. This would be a good choice for implementing a connection between Thermal Desktop and Abaqus).

Before running any linking code, two separate models with the same initial geometry were set up. Each model may have features that are not present in the other; for example, a heat lamp may exist only in the Thermal Desktop model, while a point mass might exist only in the Abaqus model. The Abaqus command line "tonastran" tool can convert an Abaqus input file to a Nastran BDF, which can be imported to Thermal Desktop with the existing mesh import tool. After both files are created, each with their relevant environments, a lookup function to relate the two sets of nodes is generated. In the cases presented in this paper, simple one-to-one lookup tables were used, because identical meshes were used in both programs.

First, an initial Abaqus file was run, establishing the starting conditions for the analysis loop. There are many methods of extracting displacements from an Abaqus analysis; in this case, the "NODE PRINT" command was used to write the displacements in plain text to the dat output file, which was read into Matlab through a Matlab subroutine. This does not require the use of an Abaqus user subroutine.

The displacements were pushed to Thermal Desktop by accessing the "DX" property of every "RcNode" object in the Thermal Desktop model. The "InitialTemp" property was also updated at this time. The Thermal Desktop file can then be run from Matlab.

\section{A. Model case}

The case for comparison with the model is Blandino's very similar work in Abaqus. ${ }^{5}$ The basic layout of the structural case, a boom under compressive load in zero gravity, is shown in Figure 8. Blandino used a subroutine to apply calculated temperatures to the nodes of a stainless steel boom with circular cross-section. Some modest differences in the analysis should be noted: Blandino chose to neglect lengthwise conduction and internal radiation in the boom, while the ray-tracing method used in the Thermal Desktop model was a Monte Carlo method, and thus does not produce identically reproducible results. A low level of noise in the thermal data can be seen throughout the analysis, and this noise was not present in Blandino's analytical treatment. 


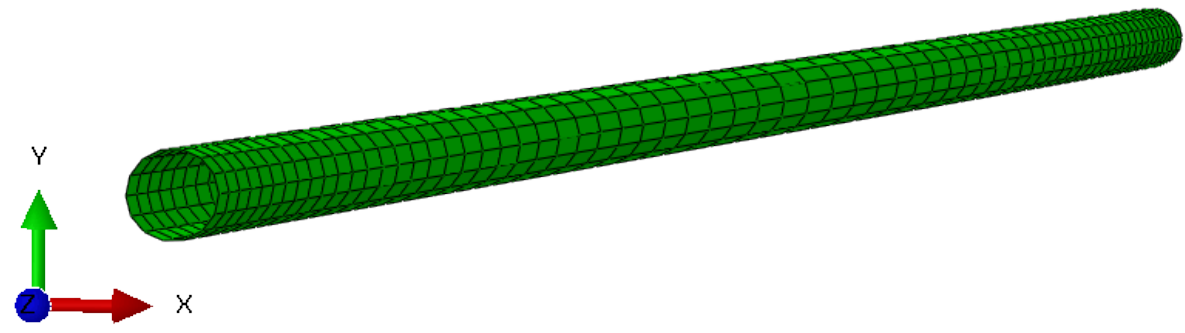

Figure 9: The mesh for the circular cross-section boom.

The dimensions of the boom are summarized in Table 1. A compressive load $P$ was applied in the axial direction $(-z)$ at levels of $P=0.31$ and $0.8 P_{c r}$, the critical buckling load. In this model, it was found that the critical buckling load for loading in the $z$-direction was $12.5 \mathrm{~N}$, slightly more than the $12.1 \mathrm{~N}$ load found by Blandino. The reason for this discrepancy has not been identified; a slightly higher stiffness was found throughout the analysis.

Table 1: Boom dimensions from Table 9.4 of Thornton, ${ }^{7}$ with additional environmental parameters from Blandino. ${ }^{5}$ The same boom dimensions were used by Blandino (Table 1).

\begin{tabular}{|cr|r|l|}
\hline \multicolumn{1}{|c|}{ Parameter } & Value & Units \\
\hline \hline Boom length & $L$ & 5.91 & $\mathrm{~m}$ \\
\hline Boom radius & $R$ & 0.01092 & $\mathrm{~m}$ \\
\hline Additional tip mass & $M_{S}$ & 1.734 & $\mathrm{~kg}$ \\
\hline Wall thickness & $h$ & 0.235 & $\mathrm{~mm}$ \\
\hline Density & $\rho$ & 7010 & $\mathrm{~kg} / \mathrm{m}^{3}$ \\
\hline Young's modulus & $E$ & 193 & $\mathrm{GPa}$ \\
\hline Coefficient of thermal expansion & $\alpha_{T}$ & $16.92 E-6$ & $\mathrm{~K}$ \\
\hline Thermal conductivity & $K$ & 16.61 & $\mathrm{~W} / \mathrm{m}-\mathrm{K}$ \\
\hline Heat capacity & $c$ & 502 & $\mathrm{~J} / \mathrm{kg}-\mathrm{K}$ \\
\hline Solar absorptivity & $\alpha$ & 0.5 & - \\
\hline Infrared emissivity & $\varepsilon$ & 0.13 & - \\
\hline Solar heat flux & $S_{0}$ & 1350 & $\mathrm{~W} / \mathrm{m}^{2}$ \\
\hline Environment temperature & $T_{\infty}$ & 0 & $\mathrm{~K}$ \\
\hline Boom initial temperature & $T_{0}$ & 0 & $\mathrm{~K}$ \\
\hline Critical buckling load (Blandino) & $P_{c r}$ & 12.1 & $\mathrm{~N}$ \\
\hline Critical buckling load (Abaqus/TD coupled) & $P_{c r}$ & 12.5 & $\mathrm{~N}$ \\
\hline Applied load & $P$ & $0.31,0.8$ & $P_{c r}$ \\
\hline
\end{tabular}

The boom, shown in Figure 9, was meshed with 14 nodes at each cross section and 68 lengthwise subdivisions, for a total of 952 elements of type S4R and 966 nodes. An identical mesh was used in both Abaqus and Thermal Desktop. All nodes of the boom root were restrained in the three translational degrees of freedom, and the boom tip nodes were tied to a single central tip node as a rigid body. The tip mass of $1.734 \mathrm{~kg}$ was applied to this tip node.

A step time of $0.2 \mathrm{~s}$ was used for the coupled analysis, while Abaqus/Standard and Thermal Desktop each used smaller increments within this time step. The Abaqus steps were run as implicit dynamic analysis with the default damping of the "TRANSIENT FIDELITY" option. Displacement results are based on the motion of the boom tip. 


\section{B. Model results}

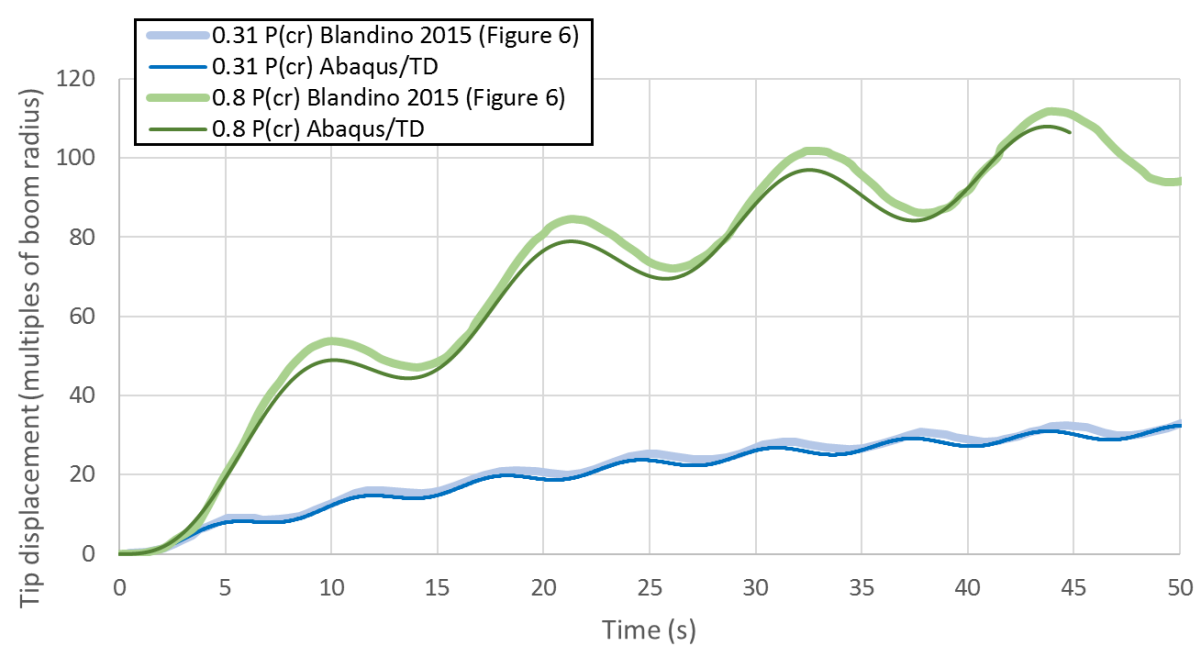

Figure 10: Comparison of results with Blandino at different levels of $P / P_{c r}$.

The model results showed good agreement with Blandino. A comparison of the resulting tip displacements, for two different levels of boom compression, is shown in Figure 10. The tip displacements differed by about $3 \%$, or about $4 \mathrm{~cm}$, in the case of $P=0.8 P_{c r}$. This was close enough to allowed continued comparisons between the Abaqus/TD method and Blandino's work.

Perhaps the most obvious question about this analysis is what difference this geometry updating capability has made. Figure 11 shows the comparison between an analysis that updated the geometry model in Thermal Desktop after every $0.2 \mathrm{~s}$ of simulated time and an analysis that did not update the geometry of the thermal model. The difference in tip displacement was negligible for this analysis case, but the effect can be seen in the boom tip temperature, shown in Figure 12. In the coupled case, the geometry in Thermal Desktop was updated to reflect that the boom tip had bent away from the sun, while in the uncoupled case, the geometry was assumed to remain constant. Thus, there was less incident light on the boom tip in the coupled case, and the boom tip was slightly cooler. In this model, the coupled analysis gives a slightly more accurate report of the boom tip temperature, without having a significant impact on the shape result.

Figure 13 shows the temperature difference between the hot side of the boom root and the hot side of the boom tip, for the coupled case with $P=0.8 P_{c r}$ compression for Blandino's analysis and the coupled Abaqus/Thermal Desktop analysis. This was another way of seeing the effect of geometry change on the thermal analysis, because the boom root heats up more quickly than the boom tip as the boom tip gradually curves away from the sun. The oscillating temperature seen in Blandino's work was a correct reflection of the oscillation of the boom tip. In this case, the oscillation was below the noise floor of the Abaqus/TD simulation. This could be improved by adjusting settings in Thermal Desktop to increase the precision of the ray-tracing Monte Carlo algorithm.

Overall, the coupled Abaqus/TD analysis produced results very similar to Blandino's. This comparison case was chosen because it was very clearly described, and the environment was simple to reproduce in Thermal Desktop. 


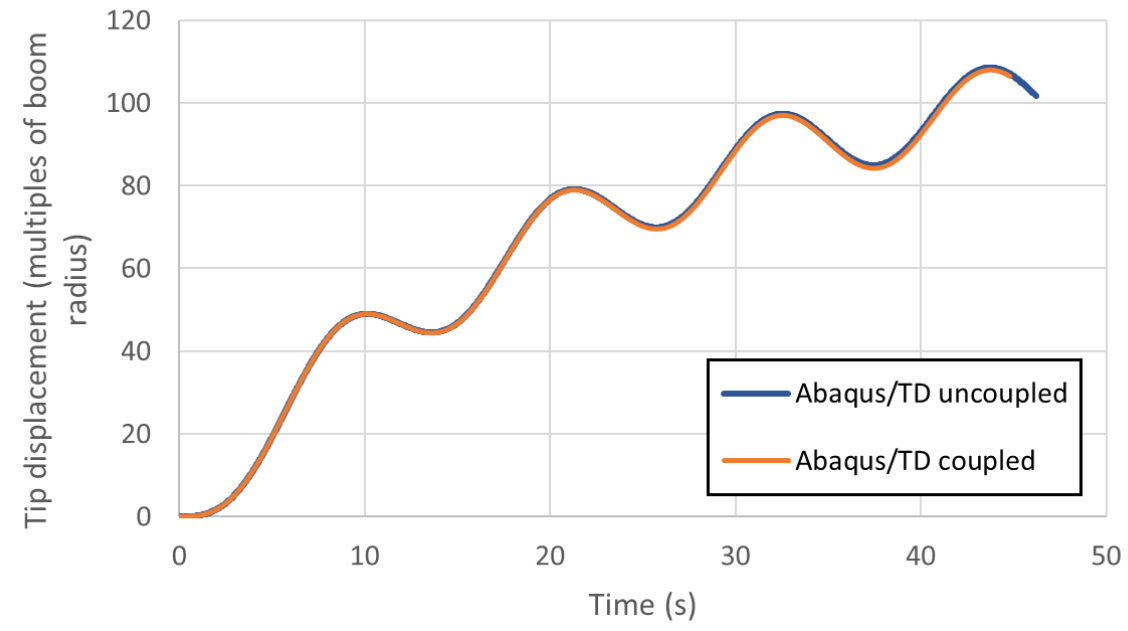

Figure 11: The boom displacement is not substantially effected by the coupling between Abaqus and Thermal Desktop in this case, where the boom tip is loaded to $0.8 P_{c r}$.

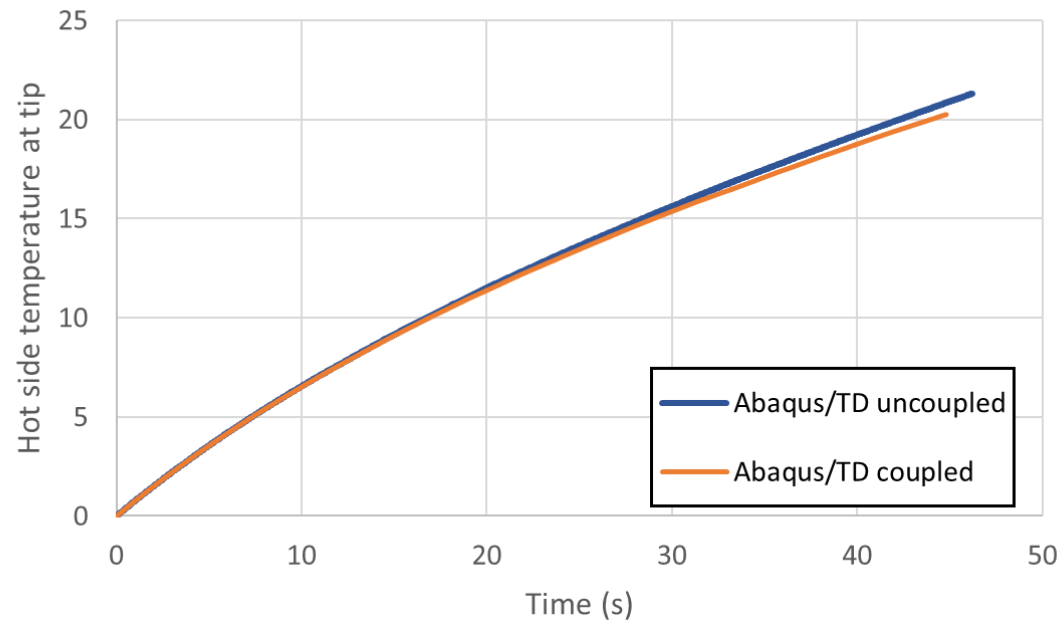

Figure 12: The effect of coupled geometry updates on the boom tip temperature. It is a small but measurable effect. The boom tip is loaded to $0.8 P_{c r}$. 


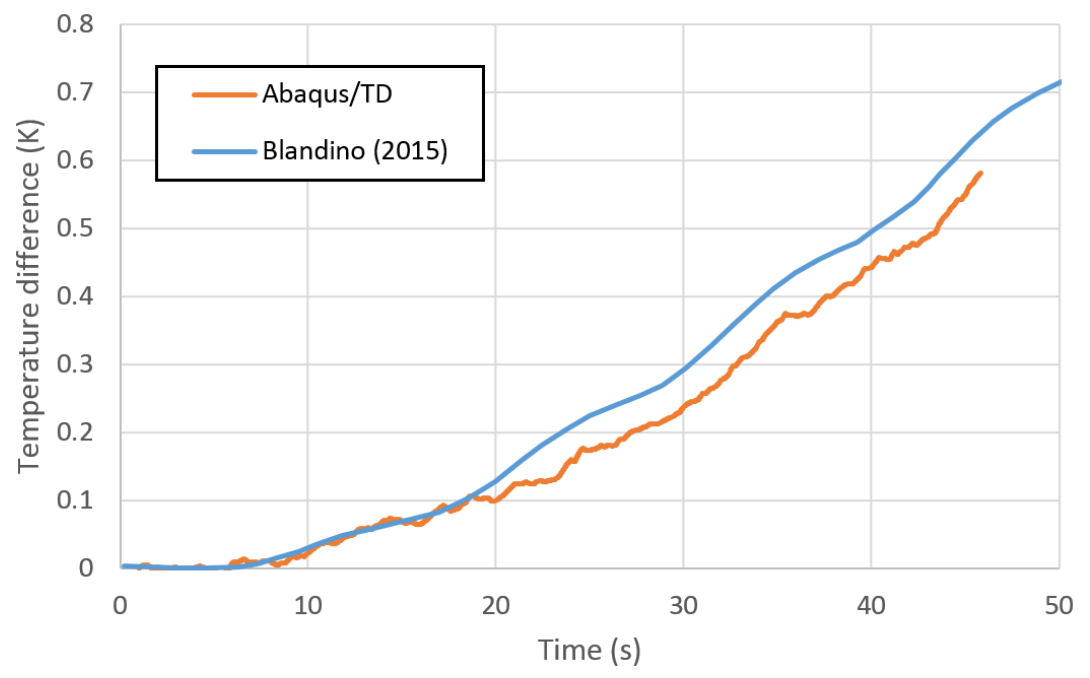

Figure 13: The difference in temperature between the hot side of the root and the hot side of the tip of the boom. The boom tip is loaded to $P=0.8 P_{c r}$.

\section{Conclusions}

It is possible to take advantage of the full capabilities of both nonlinear stress analysis software and raytracing thermal analysis software through sequential stepwise coupling. In many cases, this makes little difference in the outcome, but is it evident from experiments that some real-world cases do require a coupled approach. With some additional work in validating this method of coupled analysis, it could be reliably applied to those cases where thermal inputs create a large geometry change. As a technique, it may also simply be convenient in cases where the structural strains drive thermal results.

There is a body of experimental and analytical work on thermal flutter available for comparison with this method of coupled analysis. Thornton highlights several analytical and experimental studies of thermal flutter that are suitable for validation of this coupling. The tests conducted for the NEA Scout project are currently being modeled, but were not conducted with this type of analysis in mind, and may not have all the necessary environmental data for an accurate analysis. Further tests of TRAC booms are particularly desirable because of the dramatic effects observed in the NEA Scout tests; the TRAC boom may be a particularly good case for demonstration of structural-thermal coupling.

Several improvements to the code are possible: first, the current code uses a constant time step, which could be adjusted to respect a temperature change threshold, speeding up analysis. Second, data handling should be streamlined (for example, there is no need to retrieve temperatures from a text file; this is an available feature in the TD API). However, the most important improvement is experimental validation.

A proven method of structural-thermal coupled analysis is the eventual goal of this work, and would have been a useful tool in the early analysis of NEA Scout. In the future, with experimental correlation, it should be possible to reliably couple these analysis products, particularly in challenging cases of Cubesat-class deployables and large, ultra-lightweight spacecraft structures.

\section{Acknowledgments}

The author gratefully acknowledges the help of Gabrielle Snyder and the contributions of Erik Loper and Tiffany Lockett, co-authors on recent related conference papers. ${ }^{3,4}$ Matt Garrett of C\&R Technologies provided technical support on the Thermal Desktop API.

\section{References}

\footnotetext{
${ }^{1}$ McNutt, L., Johnson, L., Clardy, D., Castillo-Rogez, J., Frick, A., and Jones, L., Near-Earth Asteroid Scout, AIAA Space Conference, San Diego, CA, 2014
} 
${ }^{2}$ Banik, J. and Murphey, T., Triangular rollable and collapsible boom, United States patent 7,895,795, 2011

${ }^{3}$ Stohlman, O., Loper, E., Thermal deformation of very slender triangular rollable and collapsible booms, AIAA Scitech, San Diego, CA, 2016

${ }^{4}$ Stohlman, O., Loper, E., and Lockett, T., Temperature-driven shape changes of the Near Earth Asteroid Scout solar sail, 4th International Symposium on Solar Sailing, Kyoto, 2017

${ }^{5}$ Blandino, J. R., Analysis of thermal-mechanical interactions of STEM booms, AIAA Scitech, Kissimmee, FL, 2015

${ }^{6}$ McNamara, J. J. and Friedmann, P. P., Aeroelastic and aerothermoelastic analysis in hypersonic flow: Past, present, and future, AIAA Journal, vol. 49, no. 6, pp 1089-1122, 2011

${ }^{7}$ Thornton, E. A., Thermally induced vibrations, pp 343-396, in Thermal Structures for Aerospace Applications. AIAA, Reston, VA, 1996

${ }^{8}$ Beam, R. M., On the phenomenon of thermoelastic instability (thermal flutter) of boom with open cross section, NASA, Washington DC, 1969

11 of 11 